\title{
Effects of the COVID-19 Pandemic on Routine Immunization Coverage-Afghanistan, 2020
}

Samsor Rahat, BSc, MPM; Khwaja Mir Islam Saeed, MD, MSc

Afghanistan Field Epidemiology Program, Kabul, Afghanistan

Corresponding Author:

Samsor Rahat, BSc, MPM

Afghanistan Field Epidemiology Program

Wazir Akbar Khan

Kabul, 1001

Afghanistan

Phone: 93782020611

Email: $\underline{\text { samsoorrahat@gmail.com }}$

\begin{abstract}
Background: The COVID-19 pandemic has been declared the worst public health crisis in the world, with over 50 million global confirmed cases and 1,256,869 global deaths across 214 countries and territories as of November 9, 2020. Among 73 GAVI-eligible countries, 69 of them have reported COVID-19 cases; Indonesia, India, and Pakistan are the countries that comprise the highest number of cases. Afghanistan reported its first case of COVID-19 on February 24, 2020, in Herat province, which was an imported case who had a travel history from Iran. Afghanistan reported 42,092 confirmed cases and 1558 deaths as of November 9, 2020. During the COVID-19 pandemic, around 300,000 Afghans returned from Iran and Pakistan, and this overwhelmed the government's attempts to control the outbreak. There is no specific treatment for COVID-19, and no vaccine has been introduced yet. However, the World Health Organization recommends some measures in order to decrease the spread of virus transmission, such as physical distancing, avoiding gatherings, closing schools and universities, wearing masks, and home quarantine. Despite the lockdown of cities, health service delivery remains open in Afghanistan during the COVID-19 pandemic. The reporting of confirmed cases remains low; only health facilities are reporting confirmed cases and deaths. Hospitals have begun to lose staff; health staff are not only becoming ill or dying, they are also not able to work under conditions with highly contaminated environments and health hazards. The reporting of COVID-19 data has been unreliable. At first, the government only managed 100 tests per day, and the majority of people did not have access to HFs for testing, which caused the virus to start spreading throughout the community. In a recent nationwide, population-based seroepidemiological study that the Ministry of Public Health conducted on July in 9 regions of Afghanistan, around 10 million people were affected by COVID-19. The total proportion of COVID-19-positive infections was 31.5\%. The highest proportion of COVID-19-positive infections was in Kabul region (53\%). The east region had the second highest proportion of COVID-19-positive infections, which was 43\% (Ministry of Public Health, 2020). Routine immunization is a vital component in reducing morbidity and mortality and one of the top priorities for the Ministry of Public Health. The health facilities that provide EPI services remain open all over the country, but the outreach and mobile sessions have been mostly suspended due to the lockdown, and families have been unable to reach HFs due to restrictions. In Kenya, the EPI performance dropped by $20 \%$ in the second quarter of 2020. In addition, in Bangladesh, Penta-3 coverage was 50\% lower in April 2020 compared to that in the same period in 2019. As per our review on the routine immunization coverage for Pakistan, the first case of COVID-19 in Pakistan was reported on February 26, 2020, and Pakistan reported 344,839 confirmed cases and 6977 deaths as of November 9, 2020. More than 1 million children had missed vaccine doses by July 1, 2020, in Pakistan. The immunization coverage decreased by 49\% for Penta-3 during March to April 2020 when compared with that in 2019 for the same months in Pakistan. Generally, the immunization uptake dropped by more than half during the COVID-19 pandemic in Pakistan (GAVI, 2020).
\end{abstract}

Objective: The primary objective of this study is to analyze the data on how much the EPI performance dropped during the COVID-19 pandemic and to determine its impact on routine immunization coverage. The secondary objectives of this study are (1) to analyze the data from April, May, June, and July for 2019 and 2020 and compare the coverage of different antigens in this period; (2) to learn how much the coverage of routine immunization dropped due to the COVID-19 pandemic; and (3) to trend analyze Penta-3, Measles-1, and TT2+(PW) coverage from December 2019 to August 2020.

Methods: This was a descriptive analysis of secondary data that existed in the EPIMIS database at the national level. The EPIMIS database is a part of the HMIS at the Ministry of Public Health. It was developed in 2015, and it is used as a data collection 
and extraction tool at the national and provincial levels. We extracted routine immunization data from the EPIMIS database from April to July of both 2019 and 2020 for comparison. In addition, we reviewed the trend analysis conducted from December 2019 to August 2020 that compared the four months prior to the lockdown (from December 2019 to March 2020) with the COVID-19 lockdown period (from April to July 2020). We managed and analyzed the data by using Microsoft Excel 2016 and Epi Info 7.2.

Results: Data for the main EPI indicators were analyzed at the national and provincial levels. As shown in our graph, the routine immunization coverage dropped by $11 \%, 2 \%$, and $12 \%$ for Penta-3, Measles-1, and TT2+(PW), respectively, during the COVID-19 pandemic lockdown period (April to July 2020) when compared to those in 2019 for the same period. During the lockdown, the supplementary immunization activities for polio eradication nationwide, which were scheduled for May and June, were postponed as well. However, the coverage for OPV-3 decreased by $6 \%$ during the lockdown when compared with that in the same period of last year. The table shows the dropped percentages of all antigens from April to July of 2020 compared to those in the same period in 2019. This high drop in coverage was observed for TT2+(PW) and Penta-3. Moreover, coverage at the provincial level also decreased due to the COVID-19 pandemic. The highest decrease in coverage for Penta-3 and Measles-1-45\% and 43\%, respectively_was observed in Paktya province during the COVID-19 pandemic (compared to those in the same period in 2019). In addition, the highest TT2+ coverage for pregnant women was observed in Kunduz province, which dropped by $67 \%$. During the lockdown, almost $200 \mathrm{HFs}$ were closed, and the provision of fixed, outreach, and mobile sessions were limited due to COVID-19. The number of fixed sessions declined by $3 \%$, while the number of outreach sessions declined by $1 \%$ during the lockdown period. Overall, 202,408 children in fixed sessions and 24,173 children in mobile sessions were missed during the lockdown period due to COVID-19 when compared to those in the same period in 2019. This decline was observed for Penta-3, Measles-1, and TT2+(PW) coverage due to COVID-19. The trend analyses for Penta-3, Measles-1, and TT2+(PW) for December 2019 to August 2020 indicates that the coverage of routine immunization slightly declined from April to July 2020 when compared to that in the months prior to the lockdown period. Based on the trend analyses, immunization coverage started to resume in July 2020 .

Conclusions: Compared to the regional countries in which the EPI performance dropped by a high percentage, routine immunization coverage in Afghanistan dropped by $11 \%$ for Penta-3 and by $2 \%$ for Measles- 1 during the lockdown period due to COVID-19. This reveals that the Afghanistan EPI team performed better during a crisis than lower-middle-income countries. However, there is a need for proper planning to protect essential health services during emergencies. In addition, the performance of fixed and outreach sessions for EPI activities declined by $3 \%$ and 1\%, respectively, during the lockdown, which had a minor impact on routine immunization activities.

(iproc 2022;8(1):e36464) doi: $10.2196 / 36464$

\section{KEYWORDS}

immunization; COVID-19

\section{Multimedia Appendix 1}

Effects of the COVID-19 Pandemic on Routine Immunization coverage - Afghanistan, 2020. [PPTX File , $471 \mathrm{~KB}-$ Multimedia Appendix 1]

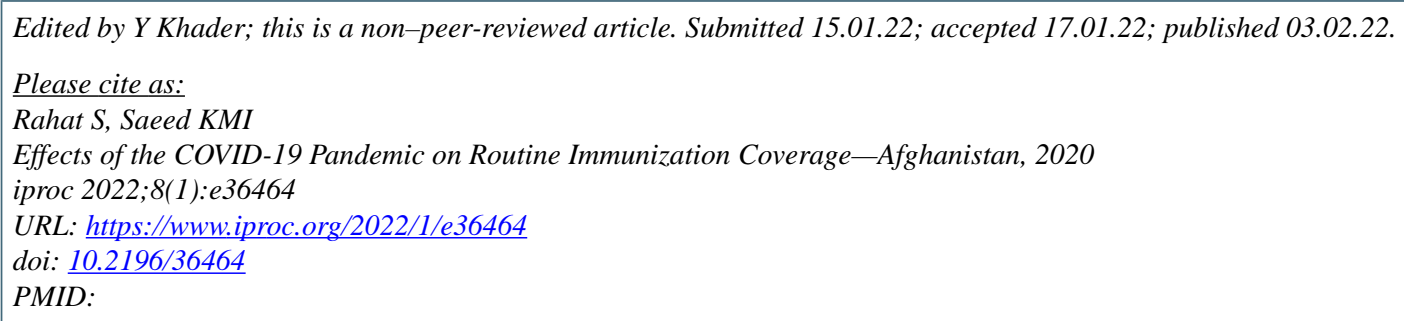

CSamsor Rahat, Khwaja Mir Islam Saeed. Originally published in Iproceedings (https://www.iproc.org), 03.02.2022. This is an open-access article distributed under the terms of the Creative Commons Attribution License (https://creativecommons.org/licenses/by/4.0/), which permits unrestricted use, distribution, and reproduction in any medium, provided the original work, first published in Iproceedings, is properly cited. The complete bibliographic information, a link to the original publication on https://www.iproc.org/, as well as this copyright and license information must be included. 